\title{
Supercontinuum Light Sources for Hyperspectral Subsurface Laser Scattering
} Applications for Food Inspection

\author{
Otto Højager Attermann Nielsen ${ }^{1}$, Anders Lindbjerg Dahl ${ }^{1}$, Rasmus Larsen ${ }^{1}$, \\ Flemming Møller ${ }^{2}$, Frederik Donbæk Nielsen ${ }^{3}$, Carsten L. Thomsen ${ }^{3}$, \\ Henrik Aanæs ${ }^{1}$, and Jens Michael Carstensen ${ }^{1}$ \\ 1 DTU Informatics, Technical University of Denmark \\ 2 DANISCO A/S \\ 3 NKT Photonics A/S
}

\begin{abstract}
A materials structural and chemical composition influences its optical scattering properties. In this paper we investigate the use of subsurface laser scattering (SLS) for inferring structural and chemical information of food products. We have constructed a computer vision system based on a supercontinuum laser light source and an AcoustoOptic Tunable Filter (AOTF) to provide a collimated light source, which can be tuned to any wavelength in the range from 480 to $900 \mathrm{~nm}$. We present the newly developed hyperspectral vision system together with a proof-of-principle study of its ability to discriminate between dairy products with either similar chemical or structural composition. The combined vision system is a new way for industrial food inspection allowing non-intrusive online process inspection of parameters that is hard with existing technology.
\end{abstract}

\section{Introduction}

The properties of a suspended materials or colloids are affected by the particle size distribution. Knowledge about particle size distribution is especially relevant for many products in the food industry, for example fat and protein particles suspended in water. The size and density distribution of particles influences parameters such as "mouth feel" and shelf life, which are important quality parameters in the food industry. In this paper we address the problem of inferring information about particle size distribution based on subsurface laser scattering (SLS). The subsurface scattering of light is affected by both the chemical and structural composition of a material 8]. Based on these properties we have designed a vision system consisting of a hyperspectral laser and a CCD camera for measuring subsurface scattering. We provide a proof-of-principle for inferring information about particle size distribution demonstrated on a number of dairy products. This system allows an opportunity for effective online monitoring of food products as well as real time process inspection, based on a non-intrusive system.

A. Heyden and F. Kahl (Eds.): SCIA 2011, LNCS 6688, pp. 327-337, 2011.

(C) Springer-Verlag Berlin Heidelberg 2011 


\section{Related work}

Other methods exist for measuring parameters affected by particle size distribution, for example rheology based on a measurement of the consistency and flow of the food product [5]. Another approach is based on measuring the scattering of a water diluted sample [14. However the water dilution can alter the particle composition of the food product. A vision system avoids the intrusive nature of these methods yet being fast and objective.

Many vision based techniques have been developed for industrial food inspection, and especially multispectral and hyperspectral methods have been successful [3/4]15]. The present system is based on a supercontinuum lightsource, filtered by and AOTF and light delivered through a single mode fiber. Together with a camera, as illustrated in Figure 1 the setup becomes a highly flexible vision system. Our setup follows the work of [1, where an SLS-system 1 devised with laser diodes is demonstrated. In their work SLS-features are correlated with the composition of milk and rheology of yoghurt. Our system is extended with multiple wavelengths.

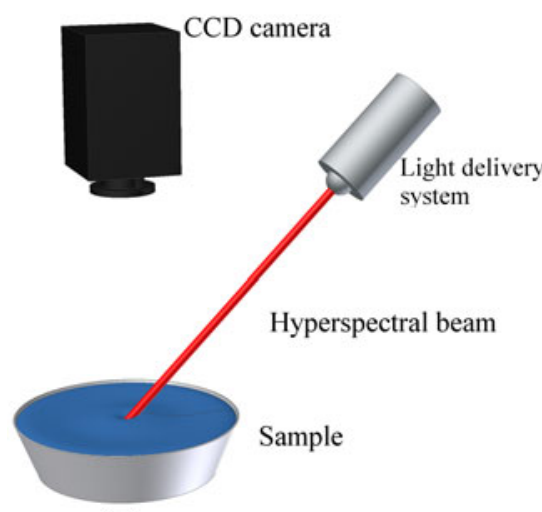

(a)

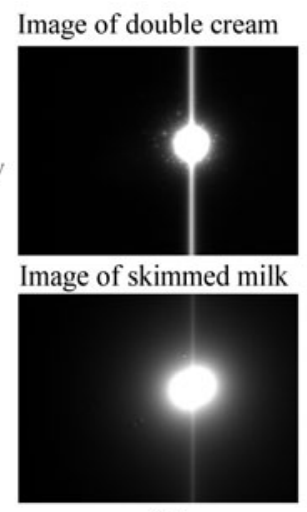

(b)

Fig. 1. (a) Illustration of our hyperspectral vision system for probing a samples SLS properties. (b) Image examples of the SLS properties of two diary products measured at $630 \mathrm{~nm}$. Both structural and chemical properties affect the appearance of the laser spot.

Hyperspectral imaging was originally developed for geology and mining based on remote sensing [11|16] and many hyperspectral analysis techniques have been developed from these problems like spectral unmixing. But hyperspectral imaging has many other useful applications including food analysis where these techniques is widely employed in both research and industry [710]15.

Hyperspectral images can be acquired by point scanning, line scanning, area scanning and single shot acquisition [15. In the point and line scanning the acquisition device or the sample, needs to be moved to obtain a hyperspectral

\footnotetext{
${ }^{1}$ http://www.videometer.com/products/products.html
} 
image, which potentially is a source of error 7]13. In the area scanning, multiple exposures are acquired from the same geometry, whereas the single shot acquires the full hyperspectral information at one exposure. Single shot has clear advantages for speed and robustness, but hyperspectral single shot technology is still under development and not ready for industrial inspection [18. The narrow spectrum for hyperspectral images are typically obtained by filtering in front of the imaging device [15, whereas we perform light filtering based on an AOTF allowing a precise control of the wavelength. In this setup we take advantage of the stable geometry with a fixed camera. The tradeoff is the need for multiple exposures - one at each wavelength. Our aim is to construct a setup where a few relevant wavelengths can be identified and subsequently used for constructing an industrial inspection system.

A very simple approach for obtaining hyperspectral images is to use a filter wheel [2, but this is restricted to a limited number of spectral bands. Tunable filters has a clear advantage in providing a flexible control of the wavelengths, but until now the use of tunable filters for food inspection has been limited 15]17. There are some examples tunable filters for quality control of food including the estimation of fruit firmness in [12] and rot of mandarins in [6] based on a LCTF (liquid crystal tunable filter). The LCTF is placed in front of the camera lens to filter the light into the camera, in contrast to our setup where the light source is filtered. The LCTF technology is capable of covering a similar spectral range as the AOTF. We have chosen the AOTF because it is appropriate with the laser beam setup that we employ.

The main contributions of this paper are:

1. A SLS computer vision setup based on a CCD camera and a hyperspectral laser obtained with an AOTF in front of a supercontinuum laser.

2. A proof-of-principle that particle size distribution can be inferred from the SLS measurements.

3. A platform for future development of hyperspectral SLS.

In the following section we will provide details of the vision system and describe how we extract relevant features. After that we show our experimental validation, and finally we discuss the obtained results.

\section{Method}

The purpose of the SLS technique is to correlate the observed image response with material properties of the measured samples. This involves design choices for the vision setup, extraction of relevant features and statistical analysis of the robustness of the measurements.

\section{Vision system}

The hyperspectral analysis is based on images acquired from the vision system shown in Figure 1 and 2. The system components are described in Table 10 The systems ability to perform hyperspectral imaging is imposed by changing 
Table 1. Hardware used in the SLS vision system

\begin{tabular}{ll}
\hline System & Device \\
\hline Supercontinuum light source & SuperK Power from NKT Photonics \\
AOTF & SpectraK Dual from NKT Photonics \\
Camera & Grasshopper CCD camera from Point Grey \\
\hline
\end{tabular}

the wavelength of the illumination light. The illumination system is based on a supercontinuum white light laser producing a quasi continues output in the range from $470 \mathrm{~nm}$ to $2400 \mathrm{~nm}$. The output is delivered in a microstructured optical fiber to an AOTF for spectral filtering of the beam. Currently the spectral filtering is supported in the range from $480 \mathrm{~nm}-850 \mathrm{~nm}$ with a spectral width growing linearly from $3.5 \mathrm{~nm}$ to $14 \mathrm{~nm}$. The final beam power output of the combined system varies as a function of wavelength from around 0.4 to $2.5 \mathrm{~mW}$, but the output is very stable over time. The AOTF is controlled by a direct digital synthesizer (DDS) which enables a fast computer controlled frequency change up to 10 times per second with an accuracy of $0.1 \mathrm{~nm}$.

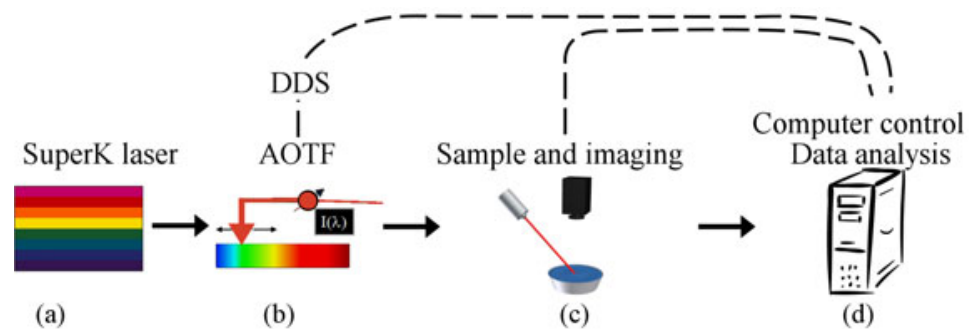

Fig. 2. Schematic illustration of the system interfaces and the scheme for generating the hyperspectral illumination system. The supercontinuum laser (a) delivers white collimated light to the AOTF, which is controlled by the DDS (b). A focused laser beam is illuminating the sample and an image is captured with a normal CCD camera (c). The entire setup is computer controlled (d).

The scattering distribution monitored from the sample will be a convolution of the scattering profile with the beams profile on the surface. Therefore, it is preferred to have a small simple beam profile. The final light delivery from the AOTF to the food sample is performed using a LMA-5 photonic-crystal fiber. The beam is collimated after the fiber using a $5 \mathrm{~mm}$ focal length lens. Simultaneously the scattering center for the hyperspectral visions system remains fixed because the LMA-5 fiber support light delivery in the full spectral range covered by the AOTF.

The scattering profile is imaged using a 16 bit CCD camera with a spatial resolution of $1600 \times 1200$ pixels. The current camera connection reduces the frame rate to $4 \mathrm{~Hz}$, resulting in a total acquisition time of about $3 \mathrm{~min}$ for a hyperspectral characterization of a sample with images from $480 \mathrm{~nm}$ to $850 \mathrm{~nm}$ with a spectral resolution of $5 \mathrm{~nm}$. 


\section{Characterization of SLS features}

The images are characterized by analysing a single profile through the scattering distribution. We have adopted a model from 1 to characterize the distributions, based on taking the logarithm to the distribution and fitted with a linear curve. An example of the data analysis is presented in Figure 3 .

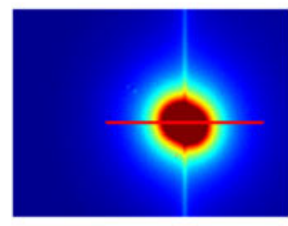

(a)

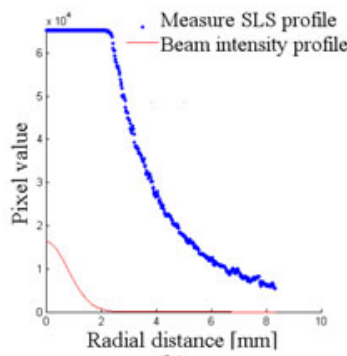

(b)

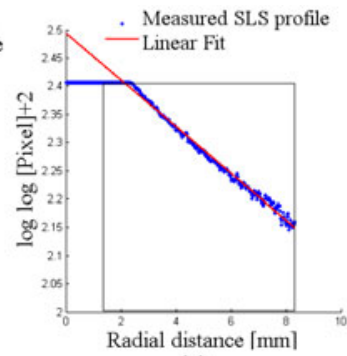

(c)

Fig. 3. Illustration of the data produced with the new vision system and the data analysis from the loglog model. (a) An image of the SLS profile in whole milk, the red trace is used in the analysis of the scattering profile (red pixels have is high intensities and blue have low). (b) Intensity through the scattering profile together with the beam profile. (c) loglog model for the scattering profile together with the linear fit. The black box indicate the range of data for the analysis.

The image from Figure 3 (a) shows a typical example of the scattering profile. It is vaguely elongated along the vertical-axis due to the beam being non orthogonal to the samples surface. In addition the images are suffering from a vertical smearing because of the heavy overexposure of the center pixels. An example of a scattering profile used and the loglog analysis of the image is presented in Figure 3. The scattering profile shows two different regimes previously presented by 9 . The interval closest to the scattering center is dominated by single scattering or diffuse reflection. In this regime the resulting scattering profile is almost proportional to the beam profile projected on the samples surface. Light rays in this part of the image have not undergone multiple scattering and do not give much information on the samples properties. The scattering pattern, which exceeds the size of the beam width, have undergone multiple scattering events and can to some extend be modeled as a diffusion process. In this range, the data with higher intensity than the background noise of the camera is fitted with a linear slope. The trace analyzed with this method results in two parameters, a slope and an offset. The offset of the loglog curve, which represents the amplitude of the SLS profile at the image center, is very system dependent. It may be possible to make the value independent of the system by calibrating the signal strength on the CCD camera. The slope of the loglog curve describes the rate of descend and is therefore a combination of the scattering properties of the sample and its absorption spectrum. A high value of the loglog slope corresponds to a narrow scattering profile. 


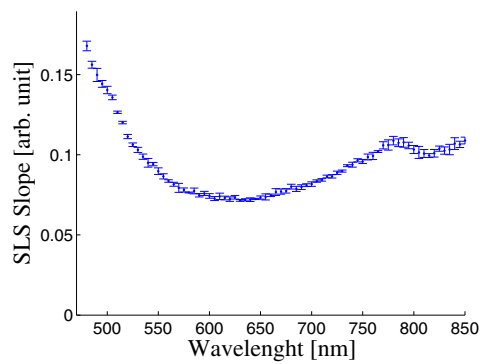

(a) Measurement of the same sample (b) repeated four times.

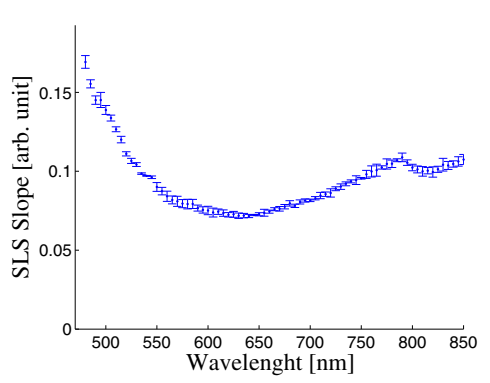

(b) Measurement of the whole procedure repeated four times.

Fig. 4. Average value and standard deviation showing the loglog slope of the scattering distribution in four measurements on the same sample of whole milk as a function of the spectral band. The figures are almost identical because whole milk is measured in both experiments - the difference is that the same milk sample was measured four times in (a) whereas four different samples were used in (b).

This form of characterization will be referred to as the loglog model. It only makes use of a limited amount of image information close to the scattering centre. The loglog model is not motivated by a physical understanding of the scattering process, but it gives a robust method that previously has been shown to correlate well with the structure of the sample [1].

\section{Reproducability}

The measurements are performed by first filling up a measuring cup to a specific height, then conduct a measurement. This procedure has two major uncertainty elements, the reproducibility of characterizing the same sample, which is a combined effect of the vision system, and a simpler uncertainty element in the way the sample and cup is positioned in the vision system. The beam is not perfectly collimated so the spot size of the laser beam may vary as a function of the samples height in the cup. A variation of the samples height will also change the size of the scattering profile measured by the camera, which is focused to fixed depth below the camera.

To estimate the vision systems robustness against variations in the characterization of a given sample, the same sample was depicted four times and analyzed with the loglog model as shown in Figure 4, The resulting average values and standard deviation is presented in Figure 4(a) Similarly the reproducibility of the measurements procedure was estimated by performing the parameters of the same product four times. The whole milk was poured into the measurement cup and analyzed with the vision system, and the results are presented in Figure 4(b). The standard deviation of the sample characterization is generally much smaller than both the amplitude and the range spanned by the slope of the full spectrum that have been analyzed. 


\section{Experiments}

Here we present the results of experiments that demonstrate the vision systems capabilities of discriminating chemical and structural variation. These measurements are the first presentation of hyperspectral characterization of the SLS properties of a material. As a proof-of-principle study a set of commercially available diary product where measured and characterized using the loglog model.

\section{Chemical composition}

The first three measurements focus on products with different chemical contents. We have chosen cream products with different fat percentage to be characterized using the SLS vision system. The different fat contents will affect the scattering profile because it increases the number of scattering centers in the sample, and thus the slope and offset of the profile. The measured profiles are presented in Figure 5 showing a generally increasing slope as a function of the fat contents.

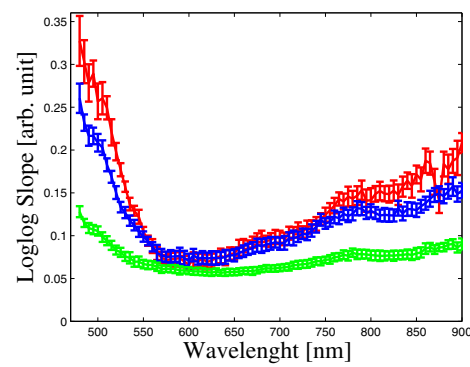

(a) Slope parameter

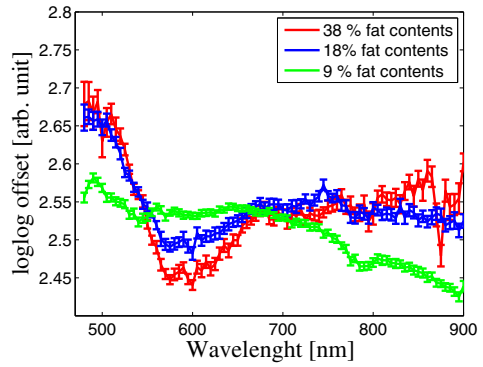

(b) Offset parameter

Fig. 5. Experiment showing the hyperspectral response for cream products differing by fat content. The fat content is especially distinguishable for low and high wavelengths for the slope parameter, whereas there is also information in the mid rage of the offset parameter.

The measurements of the scattering slope using the loglog model indicate that the largest discriminative power is found at the long and short wavelengths. The slope curves collapse in the spectral range from $\sim 530 \mathrm{~nm}$ to $\sim 700 \mathrm{~nm}$. It is seen that the changes in the slope occurs on a length scale of one hundred nanometer in this spectral range. With the new hyperspectral vision system we are able to verify this trend. However the offset still discriminates between the samples in this interval.

\section{Structural variation}

Another important parameter, that we intend to measure is the particle sizes of different components in the sample. As a first indication of this, the SLS properties of reduced fat milk was performed on conventional milk and a organic product. 


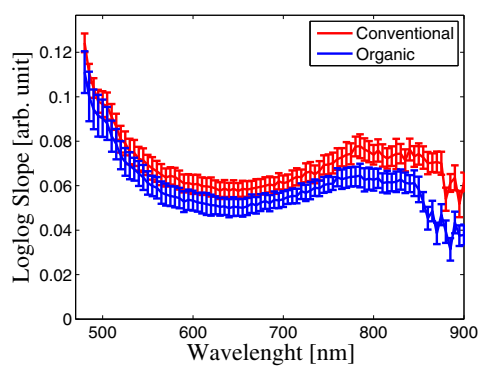

(a) Slope parameter

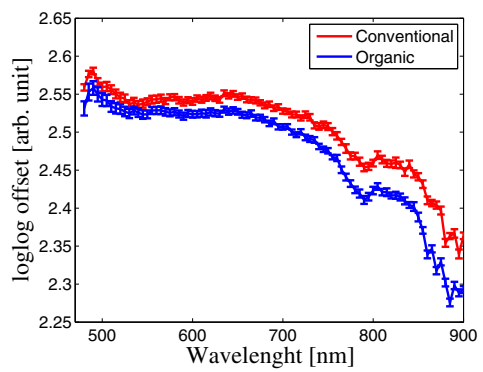

(b) Offset parameter

Fig. 6. Experiment showing the hyperspectral response for reduced fat milk differing by particle size and distribution. The organic product is unhomogenized whereas the conventional is homogenized. The homogenization process alters the size and distribution of the fat particles. Note that the difference is primarily a scale change of the SLS parameters.

These products have different particle size distributions due to the homogenization of the conventional milk, which reduces the particle size. Consequently the conventional milk has a higher density of scattering centers, but with a smaller average size. The resulting SLS response is presented in Figure 6.

The gain of hyperspectral analysis is small, but from the analysis we can choose the most discriminative wavelengths. This allows a simple but powerful method for discriminating between these products that only differ by homogenization process.

To illustrate the diversity of samples that can be examined using the new hyperspectral SLS vision system, the scattering profiles of a high particle density cream is compared to the scattering profile of fat reduced milk and yoghurt. The measured profiles are presented in Figure 7(a)

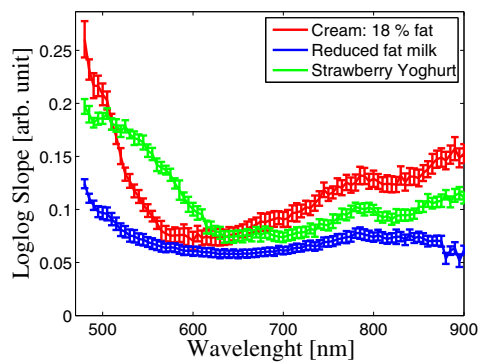

(a) Slope parameter

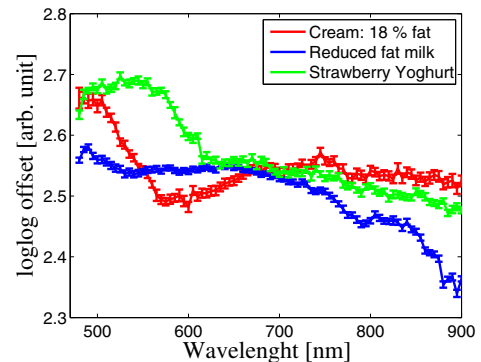

(b) Offset parameter

Fig. 7. Experiment showing the hyperspectral response for a diverse collection of products. This illustrates the large amount of information in hyperspectral SLS measurements. 


\section{Discussion}

We have demonstrated hyperspectral SLS on a number of samples and shown that we can uniquely characterize a sample using SLS parameters. Repeated studies show that the parameters only vary slightly when the data acquisition is performed several times, indicating that the system is stable over time. This is very encouraging in relation to industrial inspection of food products based on this technique.

We chose to demonstrate the hyperspectral SLS on dairy products because they contain suspended particles causing the subsurface scattering. Also a large variety of dairy products are easily available making it a good choice for demonstrating the SLS system. But the SLS technique can be used for measuring many other products - especially biological samples have subsurface scattering, making the technique widely applicable to a range of food products including meat and vegetables.

We measure a parameter based on the logarithm of the measured pixel intensity taken twice $(\log (\log (I))$, where $I$ is the image intensity), which is adopted from [1]. Empirical studies have shown that this map becomes linear, which allows a characterization based on two parameters - the slope and the intercept of the profile. The simplicity of this approach is very attractive, because it has shown to be robust and it is easy to measure. The only problem is the parameters dependence of the system, and to overcome this we need to include a calibration procedure in a future system. Further research should address the parameters characterizing a sample. One interesting application would be to directly infer particle size and distribution and another would be to measure refractive properties.

Results are reported for a single profile, so at the moment we are not utilizing the information in the entire image. A consequence is that image noise is influencing the measurements, as seen from the error bars in the graphs shown in Figure 5, 6] and 7. We could utilize more of the image to obtain higher signal to noise ratios, for example by sampling more profiles or employing a 2D model.

The characterization is based on measurements of the subsurface scattering, so we would like to optimize the system to capture as much information about the subsurface scattering as possible. The parameters governing the measurements include the beam power and profile of the laser and the exposure time of the camera. A large proportion of the light reflected close to the center of the beam is a result of single scattering or diffuse reflection. Optimally the beam profile should be as small as possible, so our relatively large profile can make the result less precise.

The beam power and exposure time have similar effect, and a large power or long exposure results in a large saturation of the depicted laser spot. This gives a high signal to noise ratio, but at the cost of information at low intensities. Low beam power or exposure will provide this information, but with lower signal to noise ratio. This tradeoff between illumination and exposure time can be accounted for by using high dynamic range, where an image is composed of multiple exposures to obtain high signal to noise ratio in both the low and high 
intensity range. In this paper we chose a single exposure, and despite this we were able to distinguish small differences like homogenized vs. non-homogenized milk.

Many of the samples are distinguishable at one wavelength, but it is important to note that this is not known in advance. Consequently our SLS system allows us to select the wavelengths with highest discriminative power, and can this way aid in constructing an optimal food inspection system, for example based on less expensive hardware like laser diodes. As a result the reconfigurable nature of the SLS system has great potential in explorative food analysis, but also in more general material characterization.

\section{Conclusion}

We have addressed the problem of inferring properties of a material from measurements of subsurface light scattering. Our contributions are $(i)$ a hyperspectral SLS (subsurface laser scattering) vision system, (ii) a procedure for characterizing the measured samples, and (iii) an experimental analysis of a number of dairy products. This explorative analysis shows a proof-of-principle of our hyperspectral SLS system for food characterization, and acts as a platform for future development.

\section{Acknowledgements}

This work was financed by the Centre for Imaging Food Quality project which is funded by the Danish Council for Strategic Research (contract no 09-067039) within the Program Commission on Health, Food and Welfare.

\section{References}

1. Carstensen, J.M., Møller, F.: Online monitoring of food processes using subsurface laser scattering. In: Advances in process analytics and control technologies APACT 2009, Glasgow, Scotland, ( May 5-7, 2009)

2. Chen, Y.R., Chao, K., Kim, M.S.: Machine vision technology for agricultural applications. Computers and Electronics in Agriculture 36(173A/191) (2002)

3. Clemmensen, L.H., Hansen, M.E., Ersbøll, B.K., Frisvad, J.C.: A method for comparison of growth media in objective identification of penicillium based on multispectral imaging. Journal of Microbiological Methods 69, 249-255 (2007)

4. Dissing, B.S., Clemmesen, L.H., Lje, H., Ersbøll, B.K., Adler-Nissen, J.: Temporal reflectance changes in vegetables. In: 2009 IEEE 12th International Conference on Computer Vision Workshops (ICCV Workshops), pp. 1917-1922 (2010)

5. Fischer, P., Windhab, E.J.: Rheology of food materials. Current Opinion in Colloid \& Interface Science (2010)

6. Gómez-Sanchís, J., Gómez-Chova, L., Aleixos, N., Camps-Valls, G., MontesinosHerrero, C., Moltó, E., Blasco, J.: Hyperspectral system for early detection of rottenness caused by Penicillium digitatum in mandarins. Journal of Food Engineering 89(1), 80-86 (2008) 
7. Gowen, A.A., O’Donnell, C.P., Cullen, P.J., Downey, G., Frias, J.M.: Hyperspectral imaging-an emerging process analytical tool for food quality and safety control. Trends in Food Science \& Technology 18(12), 590-598 (2007)

8. Jensen, H.W., Marschner, S.R., Levoy, M., Hanrahan, P.: A practical model for subsurface light transport. In: Proceedings of the 28th Annual Conference on Computer Graphics and Interactive Techniques, pp. 511-518. ACM, New York (2001)

9. Joshi, N., Donner, C., Jensen, H.W.: Noninvasive measurement of scattering anisotropy in turbid materials by nonnormal incident illumination. Optics letters 31(7), 936-938 (2006)

10. Kim, M.S., Chen, Y.R., Mehl, P.M.: Hyperspectral reflectance and fluorescence imaging system for food quality and safety. Transactions of the ASAE 44(3), 721$729(2001)$

11. Nielsen, A.A.: Spectral mixture analysis: Linear and semi-parametric full and iterated partial unmixing in multi-and hyperspectral image data. Journal of Mathematical Imaging and Vision 15(1), 17-37 (2001)

12. Peng, Y., Lu, R.: An LCTF-based multispectral imaging system for estimation of apple fruit firmness. Part 2. Selection of optimal wavelengths and development of prediction models. Transactions of the ASAE 49(1), 269-275 (2006)

13. Peng, Y., Lu, R.: Analysis of spatially resolved hyperspectral scattering images for assessing apple fruit firmness and soluble solids content. Postharvest Biology and Technology 48(1), 52-62 (2008)

14. Sacoto, P., Lanza, F., Suarez, H., Garcia-Rubio, L.H.: A novel automatic dilution system for on-line particle size analysis. In: ACS Symposium Series, vol. 693, pp. 23-29. ACS Publications (1998)

15. Sun, D.W.: Hyperspectral imaging for food quality analysis and control. Academic Press, London (2010)

16. van der Meer, F.: Imaging spectrometry for geological remote sensing. Geologie en Mijnbouw 77(2), 137-151 (1998)

17. Wang, W., Paliwal, J.: Near-infrared spectroscopy and imaging in food quality and safety. Sensing and Instrumentation for Food Quality and Safety 1(4), 193207 (2007)

18. Yasuma, F., Mitsunaga, T., Iso, D., Nayar, S.K.: Generalized Assorted Pixel Camera: Post-Capture Control of Resolution, Dynamic Range and Spectrum. Technical report (November 2008) 